\title{
Aerodynamic noise of high-speed train pantographs: comparisons between field measurements and an updated component-based prediction model
}

\author{
Xiaowan Liu $^{\mathrm{a}, *}$, Jin Zhang ${ }^{\mathrm{a}}$, David Thompson ${ }^{\mathrm{a}}$, Eduardo Latorre Iglesias ${ }^{\mathrm{b}}$, \\ Giacomo Squicciarini ${ }^{\mathrm{a}}$, Zhiwei $\mathrm{Hu}^{\mathrm{c}}$, Martin Toward ${ }^{\mathrm{a}}$, Daniel Lurcock ${ }^{\mathrm{a}}$ \\ ${ }^{a}$ Institute of Sound and Vibration Research, University of Southampton, Southampton \\ SO17 1BJ, UK \\ ${ }^{b}$ Escuela Tecnica Superior de Ingenieria y Sistemas de Telecomunicacion, Universidad \\ Politécnica de Madrid, Madrid, Spain \\ ${ }^{c}$ Aerodynamic and Flight Mechanics Research Group, University of Southampton, \\ Southampton SO17 1BJ, UK
}

\begin{abstract}
Aerodynamic noise from pantographs becomes an important source of noise from trains at high speeds. Previous studies have mostly been based on numerical predictions using computational aeroacoustic methods, which require large computing resources, or measurements conducted in a wind tunnel which cannot take all the real conditions into account. A component-based model relying on empirical constants obtained from the literature has been shown to predict aerodynamic noise from pantographs that agrees well with wind tunnel measurements. This model is extended in this paper by making use of simulation results on individual cylinders to refine the model constants and the Reynolds number dependence. In addition, allowance for the effect of incoming turbulence and cylinder aspect ratio is also extended. The updated model shows improved agreement with wind tunnel measurements, particularly at low frequencies. This model is then used to predict pantograph noise in more realistic conditions during train pass-by. The incoming flow conditions in terms of the incident flow speed, the turbulence intensity and the turbulence length scale are estimated from the literature considering the development of the boundary
\end{abstract}

\footnotetext{
* Corresponding author

Email address: Xiaowan.Liu@soton.ac.uk (Xiaowan Liu)
} 
layer along the train roof. The sensitivity of the model to these assumptions is assessed using Monte Carlo simulations. The predicted results are compared with field measurements obtained using microphone array techniques for pantograph on different operational trains. Good agreement is obtained between the predictions and the measurements in terms of the far-field noise spectra and the dependence of noise level on speed. Differences are noted between measured levels for different orientations of the pantograph which according to the model are mainly related to the distance of the pantograph from the front of the train. Keywords: Train pantograph, Aerodynamic noise, Component-based model, Microphone array measurement.

\section{Introduction}

On modern electric high-speed trains there are two main types of noise source: rolling noise and aerodynamic noise. Rolling noise is generated by wheel/rail interaction and its sound power increases in proportion to the third 5 power of the train speed $V$ [1] while the aerodynamic noise is due to interaction with air flow and contains dipole sources with sound power increasing at a rate of $V^{6}[2]$ and quadrupole sources at a rate of $V^{8}[3]$. Modern high speed trains allow operational speeds up to $350 \mathrm{~km} / \mathrm{h}$ and many studies have shown that aerodynamic noise becomes predominant above around $300-350 \mathrm{~km} / \mathrm{h}$ [4, 5].

The overall noise from the train can be obtained through pass-by noise measurements using a single microphone placed at a certain distance from the track. To identify the contributions of the different noise sources to the overall noise, however, more advanced measurement techniques are needed. Nagakura [6] used an acoustic mirror, which consists of a parabolic reflector and single or multiple microphones located at its focus, to estimate the contributions of aerodynamic noise sources generated by each part of Shinkansen trains in a wind tunnel as well as in field tests. A more generally used technique involves the use of microphone arrays together with beamforming for post-processing of the microphone signals. The beamforming analysis combines the signals from the various mi- 
crophones in the array with appropriate time delays, allowing the sound to be separated into that arriving from different directions. Various configurations of microphone array have been investigated to optimise their performance depending on the situation [7-10]. Different beamforming approaches in the signal processing have also been developed, such as direct time-domain beamforming and an indirect spatial correlation approach [8].

From microphone array measurements, a 'source map' can be produced highlighting the regions that emit high sound levels in particular frequency bands. However, it is difficult to separate the contributions of different kinds of noise source. For example, in the bogie region, when the rolling noise and the aerodynamic noise are both significant, it is difficult to separate them. Accurate quantification of different sound sources is also difficult to achieve. This is because in beamforming the sources are usually assumed to form a distribution of uncorrelated monopoles. While this assumption is reasonable for aerodynamic noise sources it is not appropriate for rolling noise, particularly as the rail is a distributed coherent line source producing a directional source field [1]. This may lead to mis-interpretation of the acoustic results when it is applied to study rolling noise [7, 11].

Examples of microphone array measurements for a TGV Duplex at different operational speeds ranging from 200 to $350 \mathrm{~km} / \mathrm{h}$ [9] showed that the main 40 aeroacoustic sources consist of the bogies, the pantograph and its recess and the front windscreen. Other microphone array measurements for different types of trains in different countries can be found in [10, 12 14]. In [10], Noh et al. presented a noise map analysis particularly for the pantograph and identified the panhead as the main source component. It is clear that the pantograph is an important source of aerodynamic noise; moreover due to its elevated position its noise cannot be efficiently shielded by noise barriers.

A number of measurements have been conducted to investigate the aerodynamic noise from pantographs. Grosche and Meier [15] measured a full-scale DSA350SEK pantograph using acoustic mirrors in a wind tunnel. They found that strong noise sources were located at the foot, the panhead and the knee 
joint. Lölgen [16] carried out wind tunnel measurements for different types of pantographs, DSA350SEK and ASP, with inflow speeds up to $400 \mathrm{~km} / \mathrm{h}$. He found that the noise spectra were characterized by the Aeolian tones associated with the vortex shedding from different cylindrical components. He identified the components contributing to the peaks using a Strouhal number relationship. Lölgen [16] also found that there were differences in the noise level between different pantograph directions, knee upstream or knee downstream. For the DSA350SEK pantograph, the noise level was 0.5-1.0 dB higher with the knee downstream; conversely for the ASP pantograph, the noise level was 3.4-6.4 dB higher with knee upstream. The noise radiated from a pantograph in both raised and lowered operating conditions was measured in a wind tunnel by Brick et al. [17. The measurements were carried out for a full-scale DSA350SEK pantograph installed inside the recess area of a mock-up roof section. They found that the noise from the raised configuration was at least $8 \mathrm{~dB}$ greater than the lowered configuration. For the raised pantograph, the noise level was found to follow a sixth power law in terms of the flow speed, which is typical of dipole-type sources.

Since the pantograph consists of a number of cylindrical components and the spectral peaks are associated with the vortex shedding from these components, 70 King and Pfizenmaier [18] conducted wind tunnel measurements to investigate the sound generation from cylinders with various cross-sections. As the cylinders in their measurements had one free end, both the peak frequency and level for circular and elliptical cylinders were found to be significantly affected by the length-to-diameter ratio when it was less than 25 , whereas the end effect was much smaller for the square cylinder. Further detailed experimental studies on the vortex shedding noise characteristics of cylinders with different cross-sections and yaw angles were carried out by Latorre Iglesias et al. [19] who measured the peak Strouhal number, noise level and directivity at Reynolds numbers up to $1.2 \times 10^{5}$ and yaw angles up to $75^{\circ}$.

Numerical techniques have also been widely applied for the prediction of aerodynamic noise from pantographs. Lee and Cho [20] predicted the aerody- 
namic noise of a two-dimensional simplified pantograph head at low speed using a combination of incompressible unsteady Computational Fluid Dynamics (CFD) analysis with the Ffowcs Williams and Hawkings (FW-H) 21] acoustic analogy. They found that the panhead section can produce low-frequency tonal noise and also high-frequency broadband noise. Ikeda and Mitsumoji [22] investigated the structure of noise sources around the panhead of a PS207 type pantograph using a combination of CFD analysis with Howe's vortex sound theory 23] applied as an acoustic analogy. They also calculated the coherence functions between the source strengths around the panhead and the noise at far-field receivers to identify the net contributions of the noise sources in the far field. Takaishi et al. 24] investigated the noise emitted from the interference between the panhead and its support based on non-compact conditions. A boundary element method was used to consider the influence of the sound reflection from the train roof. Ikeda and Mitsumoji [25] evaluated the aerodynamic noise for different configurations of the joint of the panhead and its support through CFD using Large-Eddy Simulation (LES). As the pantograph consists of a number of cylinders with different cross-sections, Liu et al. [26, 27] investigated the noise characteristics of circular cylinders and square bars at different inflow speeds by using the Delayed Detached-Eddy Simulation (DDES) method and FW-H acoustic analogy. A recent study using a combination of DDES and FW-H focusing on the aspect ratios of cuboids corresponding to small pantograph components can be found in 28.

Numerical investigations for a full-scale DSA350SEK pantograph were conducted by $\mathrm{Yu}$ et al. 29] who combined a non-linear acoustic solver analysis with the FW-H acoustic analogy to predict the aerodynamic noise. Kim et al. 30] used the Improved version of DDES (IDDES) with an improved wallmodelling capability to model a pantograph mounted in different roof cavities with rounded or chamfered edges. They achieved good agreement between their pantograph model and experimental data up to $800 \mathrm{~Hz}$ but at the higher frequencies the noise was underpredicted due to the omission of small details from the model. High-fidelity CFD methods based on LES have also been attempted 
for the simulations of a full-scale pantograph, such as in [31, 32], but these simulations require large computing resources and cannot be used routinely. Latorre Iglesias et al. 33 therefore developed a semi-empirical component-based model in which empirical constants obtained from existing measurements on cylinders were used to model the whole pantograph noise. This approach has been validated by comparisons with wind tunnel measurements [19].

The component-based model 33 is extended in this paper. The capability of the model to predict pantograph aerodynamic noise in realistic situations is also assessed by comparing with results from field measurements. The componentbased model is described briefly in Section 2. The extensions included in the updated model are introduced in Section 3. Results predicted using the current model are compared with wind tunnel measurements in Section 4. Field measurements using a microphone array have also been conducted to identify the contribution of the different noise sources of the pantograph on moving trains. These are introduced in Section 5. To correspond with the field measurements, realistic flow conditions are considered in the prediction model and the calculation procedure is adjusted to allow for the motion of the pantograph past the receiver. The prediction results are obtained in terms of the mean and standard deviation using Monte Carlo simulations over a range of realistic flow conditions. Comparisons in terms of the far-field noise spectra and the dependence of noise levels on operational speed for pantograph on different trains, including operation in both directions, are presented in Section 6 .

\section{Component-based model}

Assuming that sources from each component are not correlated, the mean square sound pressure radiated by the pantograph can be determined from the incoherent sum of the spectra of each individual strut, as follows [33],

$$
\overline{p^{2}}(\mathbf{x}, f)=\frac{\rho_{0}^{2}}{16 c_{0}^{2}} \sum_{i} \eta_{i} F_{i}(f) \frac{U_{i}^{6} D_{\operatorname{rad}_{i}}(\phi)}{R_{i}^{2}(1-M \cos \theta)^{4}}
$$


where $\rho_{0}$ is the density of air, $c_{0}$ is the speed of sound, and the subscript $i$ refers to each strut. $R_{i}$ is the distance between the noise sources and the observer. $U_{i}$ is the incident mean flow speed. $D_{\text {rad }}(\phi)=\cos ^{2} \phi$ is the directivity function where $\phi$ is the angle between the lift force vector of each component and the position vector of the observer with respect to the component position. The factor $(1-M \cos \theta)^{4}$ accounts for the convective amplification for a dipole source, where $\theta$ is the angle between the flow direction and the position vector of the observer.

For a noise source that is predominantly caused by a lift dipole, the amplitude factor $\eta_{i}$ in Eq. (1) is given by (omitting the index $i$ for clarity),

$$
\eta=S t_{\mathrm{p}}^{2} C_{\mathrm{Lrms}}^{2} l_{c} L D
$$

where $D$ is the cylinder diameter, $S t_{\mathrm{p}}(=f D / U)$ is the normalized frequency at the peak, also called the Strouhal number. $C_{\text {Lrms }}$ is the root mean square (rms) fluctuating lift coefficient, and $l_{c}$ is the spanwise correlation length, normalized by $D$.

In Eq.(1), $F_{i}(f)$ is a normalized spectrum which controls the spectral shape. It is defined as a function of frequency $f$ with $\int F(f) \mathrm{d} f=1$. Different functions are used to model the normalized spectrum for the vortex shedding peak and the broadband noise. The peak spectral shape is expressed by [33],

$$
F_{p}(f)=\frac{a_{p 1}}{\left(f_{0} / f\right)^{2}-\left(f / f_{0}\right)^{2}+a_{p 2}}
$$

155

where $f_{0}$ is the vortex shedding frequency, and the value of the parameter $a_{p 1}$ is chosen to ensure that the integral of $F_{p}(f)$ over frequency is unity. The value of $a_{p 2}$ is given by,

$$
a_{p 2}=\frac{1}{2.25}\left(\frac{B_{f}}{f_{0}}\right)^{2}
$$

where $B_{f}$ is the bandwidth, which is determined from the frequency range over 
which spectral level is within $10 \mathrm{~dB}$ of the peak.

For the broadband spectral shape, the following function is used [33],

$$
F_{b}(f)=\frac{a_{b 1}}{\left(f / f_{0}\right)^{n_{2}}+1+\left(n_{2} / n_{1}\right)\left(f / f_{0}\right)^{-n_{1}}}
$$

where the exponents $n_{1}$ and $n_{2}$ are used to control the slope of the spectrum over frequency ranges below and above the vortex shedding frequency. $a_{b 1}$ is again chosen to ensure that the integral of $F_{b}(f)$ over frequency is unity.

To predict the whole pantograph noise, the noise spectra of individual components need to be modelled first by choosing the appropriate normalized spectra and amplitude factors. These vary for different components due to differences in the cross-section (circular, elliptical, square or rectangular), the inflow turbulence level, the Reynolds number regime, the length-to-diameter ratio and the yaw angle to the flow. In the model of Latorre Iglesias et al. [33, reference values were determined based on a circular cylinder and square bar with the length-to-diameter ratio larger than 25 , in cross-flow, without inflow turbulence, and at subcritical Reynolds numbers. Various correction factors were then applied to these reference values to allow for the different effects to be considered. Based on the model of Latorre Iglesias et al. 33] some extensions have been made which are described in the next section.

\section{Extensions for determining model constants}

\subsection{The use of simulation results}

The parameters used to determine the peak and the broadband noise are listed in Table 1. For the peak noise, the values of $S t_{p}, C_{\text {Lrms }}$ and $l_{c}$ for the current predictions are obtained from the simulation results of Liu et al. [26, 27. whereas the values used by Latorre Iglesias et al. 33] were obtained from existing measurements [34. The values of $S t_{p}$ and $l_{c}$ used in the two parameter sets were similar but the values for $C_{\mathrm{Lrms}}$ showed large differences. It is recognised that $C_{\text {Lrms }}$ is extremely sensitive to experimental conditions, such as inflow turbulence levels, ratio of cylinder length to diameter and end conditions, as well 
as numerical conditions, such as grid resolution and numerical schemes for the convection terms.

There are also differences between the values used for the relative bandwidth $B_{f} / f_{0}$ in the two parameter sets. The values used by Latorre Iglesias et al. [33] 190 were obtained from noise measurements [19] in which end effects may exist. King and Pfizenmaier [18] found the end effect to be significant when a free end is used and the length-to-diameter ratio is smaller than 25. Fujita et al. 35] also indicated that, without using end-plates, there could be interference between the shear layer at the edge of the jet and the cylinder, which can disturb the two-dimensionality of the vortex shedding. The values used in the current predictions are from the simulation results [26, 27] in which periodic boundary conditions are applied in the spanwise direction and the two-dimensionality was well maintained.

Table 1: Parameters used to determine the peak noise for the reference cases based on simulations [26, 27] and experimental data [33.

\begin{tabular}{lcccccccc}
\hline & \multicolumn{4}{c}{ Peak noise } & \multicolumn{4}{c}{ Broadband noise } \\
& $S t_{\mathrm{p}}$ & $C_{\text {Lrms }}$ & $l_{c}$ & $B_{f} / f_{0}$ & $\eta_{\mathrm{b}} / \eta_{\mathrm{p}}$ & $n_{1}$ & $n_{2}$ \\
\hline \hline & \multicolumn{4}{c}{ Circular cylinder } \\
Current prediction [26, 27] & 0.19 & 0.65 & 3.2 & 0.12 & 0.016 & 3.55 & 3.55 \\
Latorre Iglesias et al. 33] & 0.19 & 0.50 & 3.0 & 0.16 & 0.057 & 1.35 & 1.35 \\
& \multicolumn{6}{c}{ Square bar } \\
Current prediction [26, 27] & 0.128 & 1.30 & 5.6 & 0.14 & 0.035 & 2.00 & 2.00 \\
Latorre Iglesias et al. 33] & 0.125 & 1.00 & 5.6 & 0.28 & 0.057 & 2.50 & 1.30 \\
\hline
\end{tabular}

The amplitude factor for the broadband noise $\eta_{\mathrm{b}}$ is determined based on its relation with the peak amplitude $\eta_{\mathrm{p}}$. The ratio of $\eta_{\mathrm{b}} / \eta_{\mathrm{p}}$ can be calculated when the difference of the overall sound pressure level ( $\triangle \mathrm{OASPL})$ between the broadband and peak noise is known, according to the relationship, $\triangle \mathrm{OASPL}=$ $10 \log _{10}\left(\eta_{\mathrm{b}} / \eta_{\mathrm{p}}\right)$. The value of $\Delta$ OASPL obtained from the noise measurements of Latorre Iglesias et al. [19] after averaging over different cross-sections and speeds was found to be $-12.4 \mathrm{~dB}$, while in the simulations of Liu et al. [26, 27. lower values of $\triangle$ OASPL were found, of about $-18 \mathrm{~dB}$ for a circular cylinder and $-14 \mathrm{~dB}$ for a square bar. This difference could be explained by end effects and 
the presence of the background noise in the noise measurements. The values of $\eta_{\mathrm{b}} / \eta_{\mathrm{p}}$ determined based on the simulations are presented in Table 1 and are used in the current predictions. The parameters $n_{1}$ and $n_{2}$ are used to determine the broadband spectral shape. As seen in Eq. (5), $n_{1}$ controls the slope of the spectral shape below the peak frequency, while $n_{2}$ controls it above the peak frequency. Higher values indicate a steeper slope. In the present study, $n_{1}$ and $n_{2}$ are obtained from the simulations in 26,27 . The values are mostly higher than those used in the predictions of Latorre Iglesias et al. 33. obtained from measurement data [19. This may be because the slope in the measurements is affected by the background noise and the shear layer noise at the edge of the jet.

The predicted noise spectra in $1 / 3$ octave bands for the reference cases are presented in Figure 1. This shows the total noise, which is the incoherent sum of the peak and broadband noise. The results predicted by using the values obtained from the simulations [26, 27] and experiments [33] listed in Table 1 are both presented. Figure 1 also includes the spectra obtained from CFD simulations from [26, 27] for comparison. For consistency, these results have been normalized by $D, L, R$ and $U$ for sound pressure level (SPL) using the following relationship,

$$
\mathrm{SPL}_{\text {normalized }}=\mathrm{SPL}-10 \log _{10} D-10 \log _{10} L+20 \log _{10} R-60 \log _{10} U
$$

For the circular cylinder, higher noise levels are predicted by Latorre Iglesias et al. 33 at frequencies above $600 \mathrm{~Hz}$ compared with the current predictions because of the lower values of $n_{1}$ and $n_{2}$. In addition, there is a difference of about $3 \mathrm{~dB}$ between the peak level in the two predictions for the circular cylinder. For the square bar, the differences between the two predictions are much smaller.

Figure 1 indicates that the model parameters used in the current prediction are appropriate to reconstruct the simulated noise spectra [26]. The difference in the OASPL is $-0.9 \mathrm{~dB}$ for the circular cylinder and $0.1 \mathrm{~dB}$ for the square bar. 

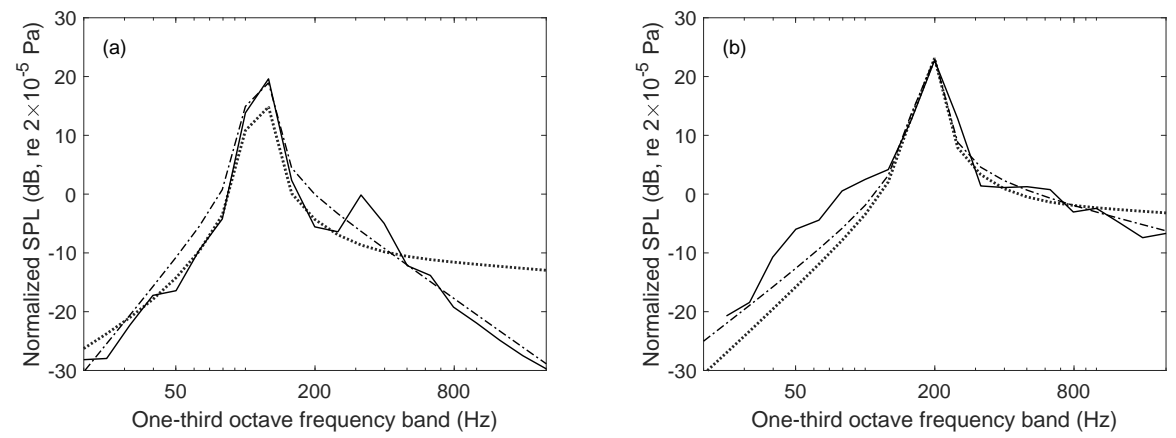

Figure 1: Comparisons between the noise spectra of the CFD simulations and the componentbased model based on different parameters of the normalized spectrum. (a) Circular cylinder at $R e=1.0 \times 10^{5} ;-$, Simulation [26] -., Current prediction, $n_{1}=n_{2}=3.55 ; \ldots$, Latorre Iglesias et al. 33], $n_{1}=n_{2}=1.35$. (b) Square bar at $R e=1.64 \times 10^{5}$; -, Simulation [27]; .- , Current prediction, $n_{1}=n_{2}=2.00 ; \ldots$, Latorre Iglesias et al. 33], $n_{1}=2.50, n_{2}=1.30$.

\subsection{Variations in the critical Reynolds number regime}

Since the pantograph components have different dimensions, and trains can run at different speeds, the corresponding Reynolds numbers vary in a wide range. In the amplitude factor $\eta$ shown in Eq. 22, the parameters $S t_{\mathrm{p}}, C_{\mathrm{Lrms}}$ and $l_{c}$ are dependent on the Reynolds number. The results are more sensitive to the Reynolds number for circular cylinders than for square bars [27]. For circular cylinders, the values of these parameters remain almost constant in the subcritical Reynolds number range but change significantly in the critical flow regime 26. 36, 37. The variations in the peak amplitude and Strouhal number with Reynolds number in the critical range were implemented in the original model [33. based on available aerodynamic measurements. The current prediction uses the numerical results from Liu et al. 26] in which simulations were carried out for part of the critical flow regime. As the value of the critical Reynolds number can vary depending on the inflow turbulence, to make the results more generally applicable the expressions for $\eta_{\mathrm{p}}$ and $S t$ are modified so that they are functions of the ratio of the Reynolds number to the critical Reynolds number, $R e / R e_{c}$

rather than Re. Here the critical Reynolds number corresponds to the transition 
point from the subcritical to critical flow regime. This allows the model to take into account changes to the critical Reynolds number, for example due to the inflow turbulence level which will be demonstrated later.

Figure 2 shows the variations of the $1 / 3$ octave band peak SPL and the Strouhal number in the critical range obtained from [26]. Here $\Delta \mathrm{SPL}=10 \log _{10}\left(\eta_{\mathrm{p}} / \eta_{\mathrm{p} 0}\right)$ is the difference between the peak SPL at a certain Reynolds number and that at the critical Reynolds number $R e_{c}$ after allowing for the dependences on $D$, $L, R$ and $U$ as shown in Eq.(6). The following relationships are used in the updated model to predict the variations of $\eta_{\mathrm{p}}$ and $S t$ in the critical Reynolds number range relative to the values obtained from the reference case (as shown in Table 1) denoted with the subscript ' 0 ',

$10 \log _{10}\left(\frac{\eta_{\mathrm{p}}}{\eta_{\mathrm{p} 0}}\right)= \begin{cases}-219.5 \log _{10}\left(R e / R e_{c}\right)+0.794 & \text { if } R e_{c}<R e \leq 1.167 R e_{c} \\ -20.5 \log _{10}\left(R e / R e_{c}\right)-12.5 & \text { if } 1.167 R e_{c}<R e \leq 2.05 R e_{c} \\ -19 & \text { if } 2.05 R e_{c}<R e \leq 3.67 R e_{c}\end{cases}$

$$
\log _{10}\left(\frac{S t_{\mathrm{p}}}{S t_{\mathrm{p} 0}}\right)=0.36 \log _{10}\left(R e / R e_{c}\right)+0.012 \quad \text { if } R e_{c}<R e \leq 3.67 R e_{c}
$$
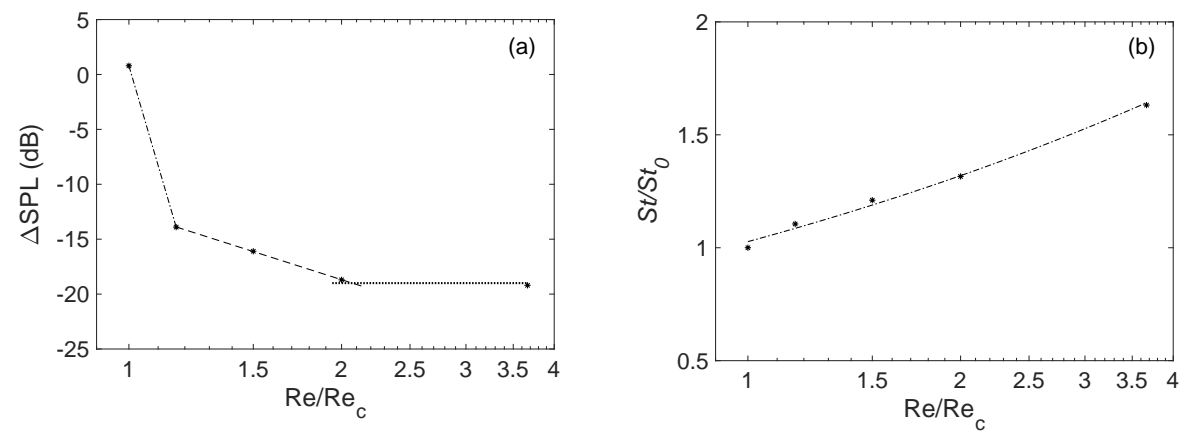

Figure 2: The variations of (a) peak SPL and (b) Strouhal number $\left(S t_{\mathrm{p} 0}=0.19\right.$ in the subcritical regime) as a function of $R e / R e_{c}$ for circular cylinders $\left(R e_{c}\right.$ is critical Reynolds number, which varies with different flow conditions, i.e. inflow turbulence intensity). 


\subsection{Effect of incoming turbulence}

The incoming turbulence can affect the peak amplitude and Strouhal number in two ways. First, the flow regime is related to the incoming turbulence. Increasing the incoming turbulence can cause the transition from the subcritical to critical flow regime to occur at lower Reynolds numbers [38]. Then the peak amplitude and Strouhal number will change as a result of the change in the flow regime. A relationship between the critical Reynolds number $R e_{c}$ and the turbulence parameter $T u$ has been proposed in 33 based on existing measurements,

$$
\log _{10}\left(R e_{c}\right)=-0.702 \log _{10}(T u)+4
$$

Here the results for $C_{\mathrm{Lrms}}$ are shown normalized to the reference value $C_{\mathrm{Lrms} 0}$, which corresponds to the value of $C_{\mathrm{Lrms}}$ listed in Table1. Figure 3 shows that, for low turbulence intensity levels, there is a sudden drop in $C_{\mathrm{Lrms}}$ from the subcritical to critical Reynolds number range. As Iu is increased, the decrease in $C_{\text {Lrms }}$ tends to become more gradual.

The following relationships, obtained according to the best fit to the data in Figure 2, are used in the updated model to account for the effect of the incoming turbulence for circular cylinders in the subcritical and critical Reynolds number 
range.

$\log _{10}\left(\frac{C_{\text {Lrms }(I u)}}{C_{\text {Lrms } 0}}\right)= \begin{cases}-0.334\left(\log _{10} I u\right)^{2}-1.3 \log _{10} I u-1.225 & \text { Subcritical Re } \\ 0.456\left(\log _{10} I u\right)^{2}+1.648 \log _{10} I u+1.488 & \text { Critical Re }\end{cases}$

Note that these relationships are applied for $I u \geq 0.004$. For $I u$ below 0.004 ,

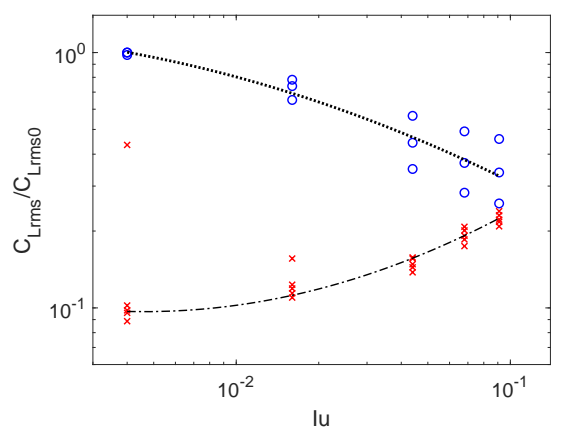

Figure 3: Effect of the incoming turbulence intensity level $I u$ on $C_{\text {Lrms }}$ for circular cylinders. $\circ$, results from Cheung and Melbourne 38 for subcritical $R e ; \times$, results from Cheung and Melbourne [38 for critical Re; ..., best fit for subcritical $R e$; -., best fit for critical Re.

280

the value of $C_{\text {Lrms }}$ is assumed to be independent of the incoming turbulence and the reference value $C_{\mathrm{Lrms} 0}$ is used.

A further extension to the original model is made to consider the effect of turbulence on the broadband noise. Available data on this are scarce. Vickery [39] measured the lift fluctuations on a square cylinder in clean flow and in turbulent flow with $I u=0.1$. The value of $C_{\mathrm{Lrms}}$ was found to reduce from about 1.32 to 0.68 due to the presence of turbulence. The percentage of spectral energy contained in a $2 \%$ bandwidth centred on the peak frequency was also measured and was found to reduce from $95 \%$ in clean flow to $82 \%$ in turbulent flow. By multiplying $C_{\mathrm{Lrms}}^{2}$ by the proportion of energy outside this bandwidth, the ratio of the broadband amplitude in turbulent flow with $I u=0.1$ to that in clean flow can be obtained as 0.955 . 


\subsection{Effect of cylinder aspect ratio}

A pantograph often contains small components, such as horn segments,

295

$10 \log _{10}\left(\eta_{\mathrm{b}} / \eta_{\mathrm{b} 0}\right)=0.0203(L / D)^{2}-0.115(L / D)-9.4, \quad$ for $\quad 3 \leq L / D<25(12)$

\section{Validation with wind tunnel measurements}

The predicted noise spectra obtained from the current model and the original model of [33] are compared with the wind tunnel measurement results [16] in 315 Figure 5 for a full-scale pantograph DSA350 in clean flow (no turbulence) with different freestream flow speeds, 230 and $350 \mathrm{~km} / \mathrm{h}$. Details of the pantograph components are given in 33. The results are presented for a receiver located 

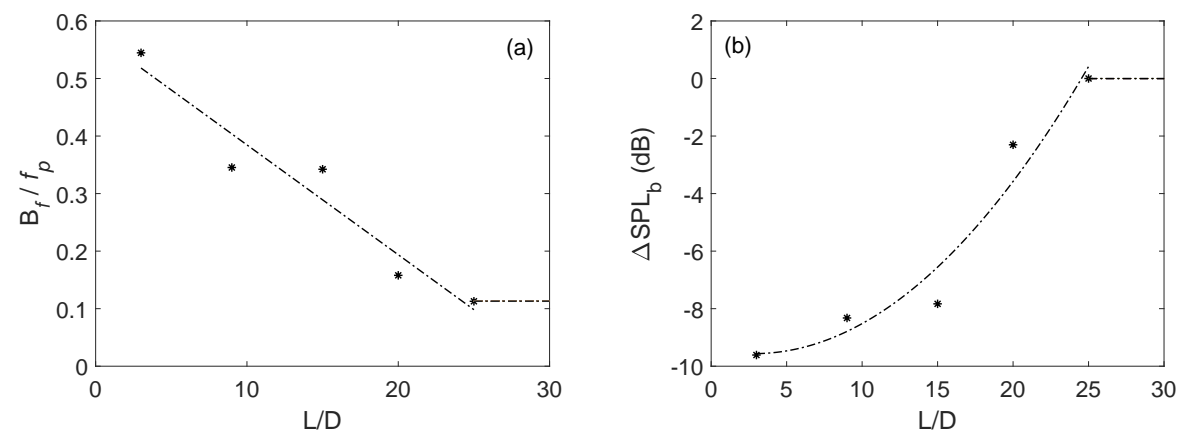

Figure 4: Variation of (a) relative bandwidth and (b) broadband SPL with cylinder aspect ratio for circular cylinders. *, measurement data from [18; -., best fit line to the data.

at a lateral distance of $5 \mathrm{~m}$ from the centre of the pantograph head normal to the flow direction. The results are given as A-weighted spectra in $1 / 3$ octave bands. Better agreement with the measured data is obtained by using the current prediction model in the region below the frequency of the first peak. This is associated with the values of $n_{1}$ and $n_{2}$. The higher values of $n_{1}$ and $n_{2}$ obtained by fitting the spectra from the CFD simulations [26, 27] used in the current predictions make the slopes of the broadband spectra steeper.

Both models overpredict the frequency of the high frequency peak. This peak is caused by circular components with small aspect ratios. Although corrections for the aspect ratio have been included in the model, the dependence of the peak frequency on the aspect ratio was determined in [33] from limited data, especially for $L / D<10$. In addition, the levels at this peak in the current prediction are slightly lower than those found by Latorre Iglesias et al. 33. This is because the current model includes a reduction in the values of $C_{\mathrm{Lrms}}$ due to high turbulence levels for circular cylinders in the subcritical Reynolds number range (see Section 3.2), which are used for components that are in the wake of others. The results of both models agree well with the measurement in the middle frequency range (between 200 and $2500 \mathrm{~Hz}$ at $230 \mathrm{~km} / \mathrm{h}$ or $315-4000$ $\mathrm{Hz}$ at $350 \mathrm{~km} / \mathrm{h}$ ) with a maximum difference of less than $4 \mathrm{~dB}$. 

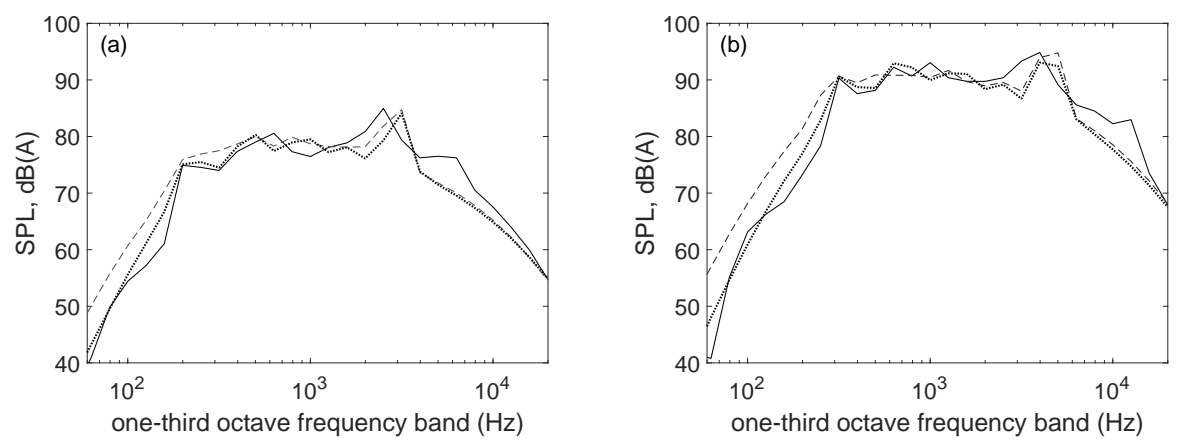

Figure 5: Comparisons of A-weighted noise spectra between the predictions and the measurements from [16] for a full-scale pantograph DSA350SEK at (a) $230 \mathrm{~km} / \mathrm{h}$ and (b) $350 \mathrm{~km} / \mathrm{h}$. -, measurements [16] ..., current prediction; - -, the prediction of Latorre Iglesias et al. 33.

\section{Microphone array measurement}

Measurements were made during train passages using the microphone array as shown in Figure 6. This was located $10 \mathrm{~m}$ from the centre of the track. The design of this microphone array includes 90 microphones (see [40]) mounted on a carbon fibre frame, positioned in the arrangement shown in Figure 6(b). This array has a $3-\mathrm{dB}$ resolution (the diameter of the main lobe $3 \mathrm{~dB}$ below the peak) of $2.3 \mathrm{~m}$ at $400 \mathrm{~Hz}$ and $4.6 \mathrm{~m}$ at $200 \mathrm{~Hz}$, which can be considered as the lower limits of the usable frequency range for the panhead and the whole pantograph. The upper limit of the usable frequency range is the $4000 \mathrm{~Hz}$ one-third octave band. Two types of train were recorded. The pantographs on them are named pantograph 1 and pantograph 2 respectively. Results for pantograph 1 are available at $273 \mathrm{~km} / \mathrm{h}$ in both directions while for pantograph 2 measurements were made for speeds between 160 and $230 \mathrm{~km} / \mathrm{h}$ and in both directions. Some of these measurement results have been presented in [41].

The beamforming techniques for moving sources, such as the ones applied to aircraft fly-over measurements and vehicle pass-by measurements, are often based on the assumption that all the sources are uncorrelated monopoles [11, 12, 42. The beamforming algorithm implemented for this study also makes use of this assumption. A virtual scanning grid is defined over the surface of 


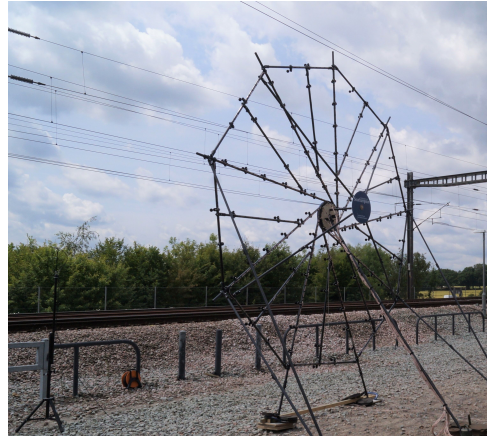

(a)

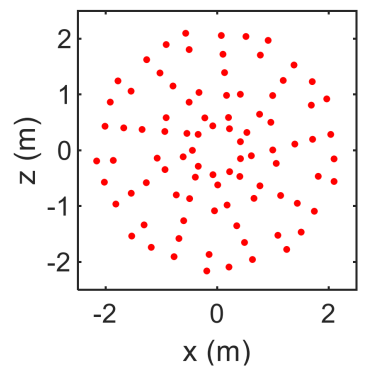

(b)

Figure 6: Microphone array setup, (a) field measurements; (b) microphone positions.

the moving train, identifying all the potential sources, and each point on the grid is 'tracked' over a certain length. This length is set to be $3.2 \mathrm{~m}$ in this study. Appropriate delays, based on the relative distance between the potential locations of the sources on the moving train and the array microphones, are applied to the measured signals to provide estimates of the source strengths at those locations. The results can be shown as a colour map (beamforming output) that can be superimposed on an image of the train. The regions with colours corresponding to higher intensity (main lobes) identify sources with higher noise contributions. In applying delays to the measured signals the Doppler effect is removed and the beamforming outputs are therefore shown in a frame of reference moving with the train. The contribution from each source can be quantified by integrating the beamforming output over the area enclosing the sources of interest.

The quantification approach adopted in this work is based on the Source Power Integration (SPI) method [42]. It can be divided into the following steps. (1) The pass-by of a monopole is simulated over the length of a single car to obtain a frequency-dependent compensation factor. This will be used to relate the beamforming output to the SPL contribution at a reference microphone, set at the centre of the array. (2) The various sources on the map are then identi375 fied and enclosed in a certain pre-defined area centred at the peak of the main lobe of that source. (3) A frequency-dependent result for each of the identi- 
fied sources is obtained by integrating the acoustic map over the corresponding source areas. (4) Finally, the compensation factor from (1) is applied to the integrated beamforming result to give the spectrum of each source. The effect of the compensation factor is to rescale the noise spectra estimated from the beamforming output so that they represent sound pressure spectra at the array centre averaged over the length of a single car. More details on the quantification algorithm are given in [40].

\section{Comparisons with field measurements}

385

The incoming flow conditions for the components in each group are estimated considering the development of the boundary layer on the train roof, which will be described in the next section.

\subsection{Estimations of incoming flow conditions}

400

When applying the prediction model to this situation, realistic incoming flow conditions, such as the mean flow velocity, the turbulence intensity and the streamwise turbulence length scale, for each component of the pantograph need to be taken into account. These parameters vary with distance from the front of the train as well as height above the train roof as a result of the boundary layer 

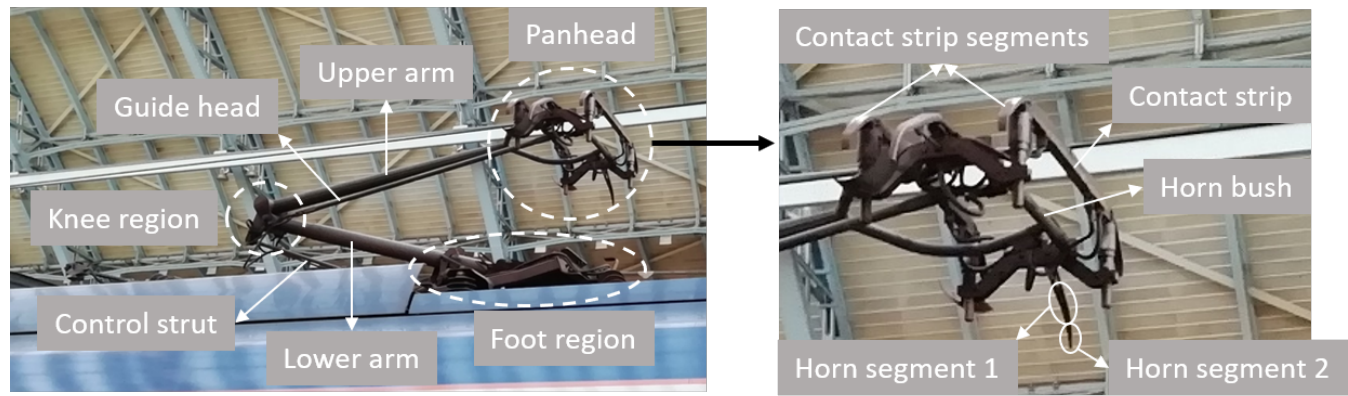

Figure 7: The pantograph with some key components identified.

Table 2: The classification of the pantograph components according to their relative positions. The components in each group are assumed to have the same incoming flow conditions.

\begin{tabular}{c|c|c}
\hline & \multicolumn{2}{c}{ Knee upstream } \\
\hline \hline $\begin{array}{c}\text { Above } \\
\text { knee joint }\end{array}$ & $\begin{array}{c}\text { Upstream contact strip, Horn } \\
\text { bush, Horn segments, Upper arm }\end{array}$ & $\begin{array}{c}\text { Downstream region } \\
\text { hownstream contact strip, Guide }\end{array}$ \\
\hline $\begin{array}{c}\text { Below } \\
\text { knee joint }\end{array}$ & Control strut & Lower arm \\
\hline \multicolumn{2}{c}{ Knee downstream } \\
\hline $\begin{array}{c}\text { Above } \\
\text { knee joint }\end{array}$ & $\begin{array}{c}\text { Upstream contact strip, Horn } \\
\text { bush, Horn segments, Guide head }\end{array}$ & $\begin{array}{c}\text { Downstream contact strip, Upper } \\
\text { arm }\end{array}$ \\
\hline $\begin{array}{c}\text { Below } \\
\text { knee joint }\end{array}$ & Lower arm & Control strut \\
\hline
\end{tabular}

development along the train roof. This subsection describes the estimations of these parameters in terms of their mean and standard deviation which are used as input in Monte Carlo simulations to assess the sensitivity of the model to these assumptions.

The values for the incoming flow parameters are estimated based on published measurements for a variety of train types at full scale and model scale with no cross wind [43 46]. The model scale results have been transformed to the equivalent full scale ones. Since the available data for the train roof are rare, data measured in the boundary layer at the train side were also included. This is acceptable for the panhead region as it is located in the upper part of the 415 boundary layer and well away from the train nose region (several vehicle lengths 
from the front of the train) where the difference in the flow fields between the train side and train roof can be considered small [44, 46].

The mean flow velocities along the train surface obtained from the measurements in the literature are shown in Figure 8. In the figure, $x$ is the distance from the front of the train, $U_{\infty}$ the operational speed of the train, and $U_{0}$ the local mean flow velocity relative to the train. The mean flow velocities were measured at different distances from the train surface. An interpolation based on the $1 / 7^{\text {th }}$ power velocity profile law [47,

$$
\frac{U_{0}}{U_{\infty}}=\left(\frac{y}{\delta}\right)^{\frac{1}{7}}
$$

was applied to obtain results corresponding to the range of panhead heights considered here. In this expression $y$ is the height above the train roof and $\delta$ the boundary layer thickness. Eq. 13 applies to the components immersed within the boundary layer $(y<\delta)$. For components outside the boundary layer $(y>\delta), U_{0}$ is taken to be equal to $U_{\infty}$. The $1 / 7^{\text {th }}$ power law was chosen for the interpolation as it is simple and effective to apply, and it can also approximate the velocity profile in many flows in practice. To apply this power law, the boundary layer thickness is assumed to be equal to that in the measurements from the literature [43, 46]. It can also be obtained by using the turbulent flat plate boundary layer formula or more advanced formulas considering pressure gradient [48. The velocity profile estimated by this power law has been compared with the measurement data from the literature, showing good agreement ${ }_{430}$ in the upper part of the boundary layer.

Through the interpolation, a greater range of data for the mean flow velocity at the considered locations is obtained. In addition, instead of depending on a fixed $x$, the data within one vehicle length including the pantograph are used. These allow a statistical distribution to be formed. The mean and standard 435 deviation determined from this distribution for the incoming flow velocity are listed in Table 3 for pantograph 1 with both knee orientations. The panhead height and the distance from the front of the train are also included in Table 3 


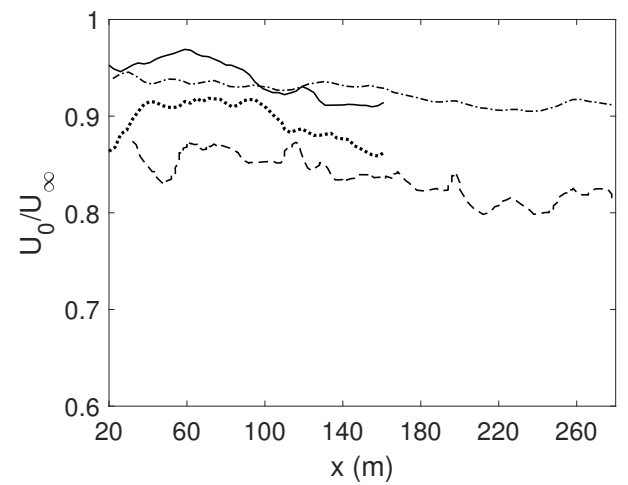

Figure 8: The normalized mean flow velocity above the train surface from different measurements in the literature. ..., Soper at al. [46] for a model scale freight train with full loading configuration at $0.7 \mathrm{~m}$ above the train roof; - , Soper at al. 46 same train at $1.8 \mathrm{~m}$ above the train roof; --, Sterling et al. 45] for a full scale ICE1 at about $0.6 \mathrm{~m}$ away from the train side; -., Sterling et al. 45 same train at about $1.4 \mathrm{~m}$ away from the train side.

Table 3: Pantograph 1 configurations and the estimated incoming flow conditions for the components in the upstream region above the knee joint. Both mean and standard deviation (in parenthesis) for each incoming flow parameter are reported.

\begin{tabular}{|c|c|c|c|c|c|}
\hline Knee orientation & $\begin{array}{l}\text { Distance } \\
(\mathrm{m})\end{array}$ & $\begin{array}{l}\text { Panhead height } \\
\text { above roof }(\mathrm{m})\end{array}$ & $U_{0} / U_{\infty}$ & $I u$ & $\begin{array}{l}L_{x} \\
(\mathrm{~m})\end{array}$ \\
\hline Knee downstream & 280 & $0.7-0.8$ & $\begin{array}{c}0.84 \\
(0.03)\end{array}$ & $\begin{array}{c}0.07 \\
(0.02)\end{array}$ & $\begin{array}{c}4.8 \\
(2.0)\end{array}$ \\
\hline Knee upstream & 100 & $0.7-0.8$ & $\begin{array}{c}0.88 \\
(0.03)\end{array}$ & $\begin{array}{c}0.07 \\
(0.02)\end{array}$ & $\begin{array}{l}4.8 \\
(2.0)\end{array}$ \\
\hline
\end{tabular}

The turbulence intensity $I u$ above the train surface has been found to be more or less constant along the train [4]. The levels decrease with the distances from the train surface and were found to be of the order of 0.05 to 0.1 for turbulent boundary layers on the train side [43. For this parameter, a mean of 0.07 and standard deviation of 0.02 are assumed for pantograph 1 with both knee orientations, which are consistent with the measured data.

${ }_{445}$ The streamwise integral length scale $L_{x}$ for a model scale freight train with full loading configuration, exhibiting similar flow development to a passenger train with inter-carriage gaps, was found to be about $4.8 \mathrm{~m}$ at a distance of 0.7 $\mathrm{m}$ above the train roof and about $7.0 \mathrm{~m}$ as the distance was increased to 1.8 
$\mathrm{m}$ [46]. Baker [43] indicated that the integral length scale was of the order of $20 \%$ of the length of an individual carriage. The value for this parameter varies little for different types of passenger trains [43, 45]. The mean and standard deviation listed in Table 3 for this parameter are consistent with these results.

The pantograph geometry used for pantograph 1 is also applied to pantograph 2. The main differences between these two cases are the distance of the pantograph from the front of the train and the panhead height above the train roof. The configurations for pantograph 2 within the train for both orientations are listed in Table 4. Also given are the corresponding incoming flow velocity $U_{0}$, turbulence intensity $I u$ and streamwise integral length scale $L_{x}$ which are estimated in the same way as for pantograph 1 . Compared with the first pantograph, this one had a greater panhead height above the train roof and was located closer to the front of the train. Consequently, higher values of mean flow velocity and integral length scale, and lower turbulence intensity levels are used.

Table 4: Pantograph 2 configurations and the estimated incoming flow conditions for the components in the upstream region above the knee joint. Both mean and standard deviation (in parenthesis) for each incoming flow parameter are reported.

\begin{tabular}{|c|c|c|c|c|c|}
\hline Knee orientation & $\begin{array}{l}\text { Distance } \\
(\mathrm{m})\end{array}$ & $\begin{array}{l}\text { Panhead height } \\
\text { above roof }(\mathrm{m})\end{array}$ & $U_{0} / U_{\infty}$ & $I u$ & $\begin{array}{l}L_{x} \\
(\mathrm{~m})\end{array}$ \\
\hline Knee downstream & 100 & $1.0-1.2$ & $\begin{array}{c}0.90 \\
(0.03)\end{array}$ & $\begin{array}{c}0.05 \\
(0.02)\end{array}$ & $\begin{array}{c}6 \\
(2.0)\end{array}$ \\
\hline Knee upstream & 40 & $1.0-1.2$ & $\begin{array}{c}0.93 \\
(0.03)\end{array}$ & $\begin{array}{c}0.05 \\
(0.02)\end{array}$ & $\begin{array}{c}6 \\
(2.0)\end{array}$ \\
\hline
\end{tabular}

Having determined the statistical distribution (a normal distribution is assumed here) with appropriate mean and standard deviation, a number of random values can be generated from the distribution for each input parameter. These values are used in the prediction model for the upstream components above the knee joint. For the components in other groups, as listed in Table 2 . the incoming flow conditions for both pantograph 1 and 2 are assumed as:

- Above the knee joint and in the downstream region: the flow interaction between the upstream and downstream components is considered to be 
insignificant as the separation distance is sufficiently large. However, the incident flow speed will be reduced so an incident speed of $0.85 U_{0}$ and a turbulence intensity level of 0.1 are assumed according to the measured downstream flow characteristics of a square cylinder from [49, 50, which are also consistent with the simulation results from Kim et al. 30] for a complex pantograph model. $L_{x}$ is assumed to be equal to the dimensions of the cross-sections of the upstream components.

- Below the knee joint and in the upstream region: as the components are located closer to the train surface than those above the knee joint, a lower incident flow speed of $U_{0}=0.8 U_{\infty}$ and a high intensity level of $I u=0.1$ are assumed according to the flow characteristics of the turbulent boundary layer on the train surface that have been introduced above.

- Below the knee joint and in the downstream region: again, no flow interaction with the upstream components is considered. Values of $U=0.85 U_{0}$ and $I u=0.1$ are assumed, where $U_{0}$ is now the incident flow speed for the upstream components below the knee joint.

The predictions for each pantograph case are carried out based on 4000 combinations of $U_{0}, I u$ and $L_{x}$ with 20 values for $U_{0}$ and $I u$, and 10 for $L_{x}$. The results will be presented in terms of the mean and standard deviation in each frequency band.

\subsection{Calculations for a moving pantograph}

To allow comparisons between the results of the model and those obtained from the experimental quantification procedure, in which the pantograph is considered as a source moving over the length of a single car, the noise is calculated for different locations of the pantograph within this length, see Figure 9 , and the average level is determined. To be consistent with the array measurements, the sound pressure at the array centre is obtained by taking the average over different heights within the range -2 to $2 \mathrm{~m}$ relative to the centre. The pre- 

microphone heights

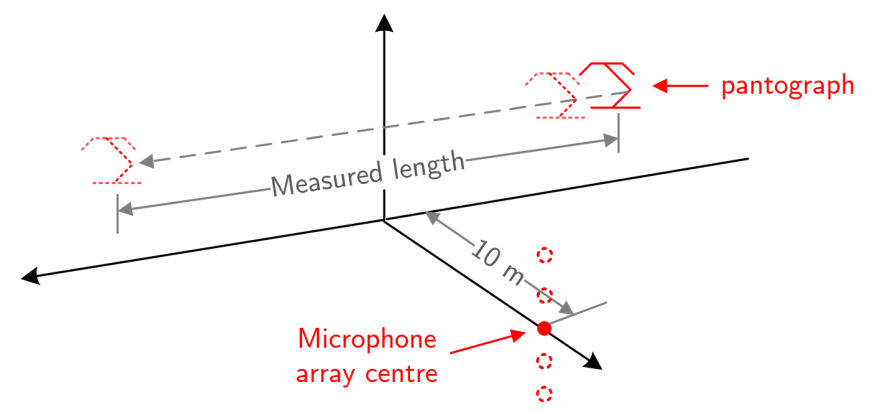

Figure 9: Calculations in the model are performed for pantograph at different locations

The measured and predicted results are presented for the pantographs mounted on different trains. Comparisons in terms of noise spectra are presented for pantograph 1 at $273 \mathrm{~km} / \mathrm{h}$ and pantograph 2 at $226 \mathrm{~km} / \mathrm{h}$. In addition, for pantograph 2 measurements at different speeds are available which allow comparisons for the dependence of noise level on speed.

\subsection{Results for pantograph 1}

Noise maps are shown in Figure 10 for the first pantograph in two different orientations, knee joint downstream and upstream. These correspond to the frequency range $400-4000 \mathrm{~Hz}$. The light regions in the noise map indicate the sound sources with high levels. For both configurations, the predominant source is found at the panhead. The knee joint region is also found to be important although the level is lower than that at the panhead. The sound levels of both the panhead and the knee joint appear to increase for the knee upstream configuration compared with the knee downstream one.

The measured A-weighted noise spectra in $1 / 3$ octave bands for the panhead, knee joint and whole pantograph for both configurations are presented in Figure 11. These correspond to the integrations over non-overlapping square regions around these components. The dimensions of these regions are chosen as $1.3 \mathrm{~m}$ and $1.2 \mathrm{~m}$ respectively for the panhead and the knee joint which avoids 
including the foot region and recess. A threshold of $12 \mathrm{~dB}$ as used in [51] is implemented for the map integration to exclude the influence caused by undesired sidelobes. The spectrum for the whole pantograph was obtained by taking the incoherent sum of the spectra of the panhead and the knee joint. It is seen that the panhead dominates the whole frequency range for both configurations and the contributions of the knee joint are around $8 \mathrm{~dB}$ lower. When the knee is downstream, the spectrum of the whole pantograph shows a slight peak at $800 \mathrm{~Hz}$, while this peak is not found for the knee upstream case as the levels above $800 \mathrm{~Hz}$ becomes higher. Similar increases between 800 and $2000 \mathrm{~Hz}$ are also found for both the panhead and the knee joint. Figure 11 also shows the OASPLs for the whole pantograph, the panhead and the knee joint for both pantograph orientations. The OASPL of the knee joint (for knee downstream case) is indicated as $\mathrm{A} d \mathrm{~B}(\mathrm{~A})$; the other OASPL values are shown relative to this value. The OASPL of the whole pantograph for knee upstream is about 2 $\mathrm{dB}(\mathrm{A})$ higher than that for knee downstream.

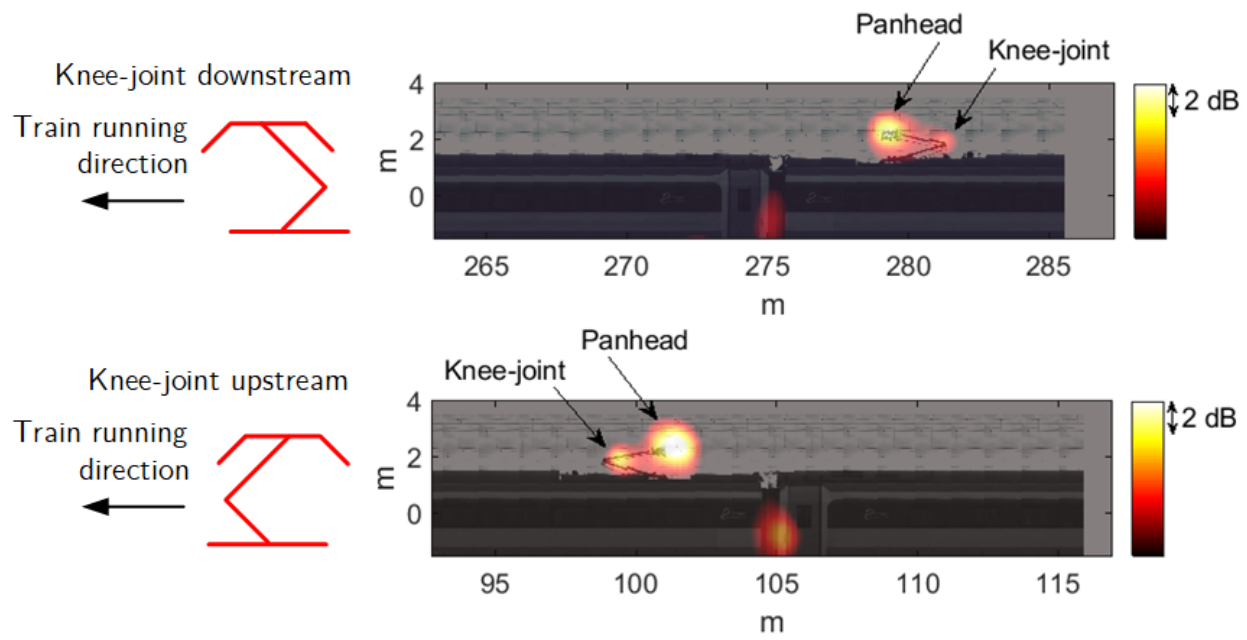

Figure 10: Noise maps over 400 to $4000 \mathrm{~Hz}$ for pantograph 1 at $273 \mathrm{~km} / \mathrm{h}$ with knee-joint downstream (Top) and upstream (Bottom) (see also in [41]). Colour maps are to the same scale.

The predicted noise spectra are shown in Figure 12 in terms of the mean and a range of $+/-$ one standard deviation in each one-third octave band, obtained 

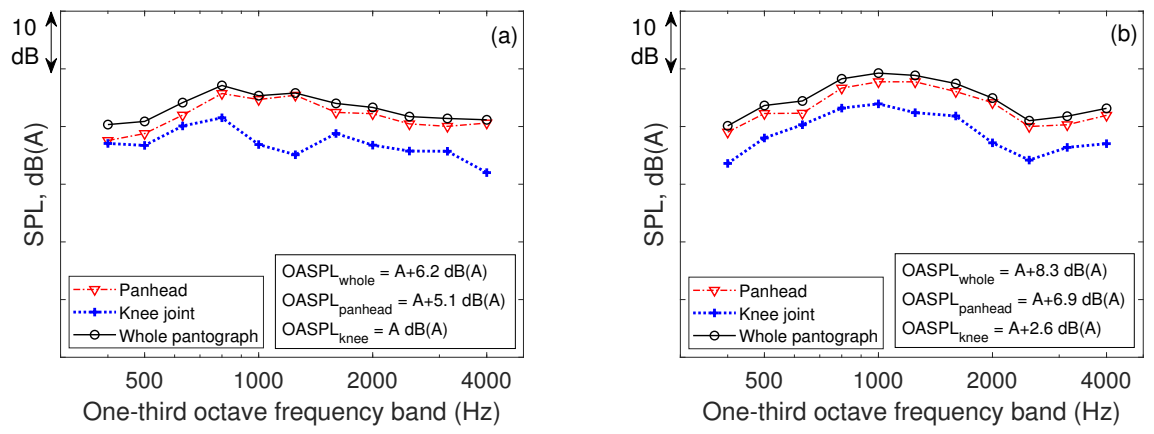

Figure 11: Measured noise spectra of two source regions, the panhead and the knee joint for pantograph 1: (a) knee joint downstream; (b) knee joint upstream. Graphs are to the same scale. OASPL values are shown relative to the value for the knee joint (for knee downstream orientation).

based on 4000 combinations of $U_{0}, I u$ and $L_{x}$. The standard deviation range is represented by the error bar. The contributions from the knee joint, pantograph foot region and recess are not included in the current prediction model. The flow developments in these regions are complex and the corresponding noise generation cannot be simply considered as vortex shedding noise. Therefore, these results are compared with the measurement results for the panhead region in Figure 12 .

Good agreement is obtained with the measurement results over the considered frequency range $200-4000 \mathrm{~Hz}$, with a maximum difference of about 3 $\mathrm{dB}$ for the knee downstream and $5 \mathrm{~dB}$ for the knee upstream. The maximum differences for both orientations occur in the region 315 to $630 \mathrm{~Hz}$ where the standard deviations are larger indicating that the noise levels are more sensitive to the incoming flow conditions than at other frequencies.

It was observed in Figure 11 that the measured levels were increased by around $2 \mathrm{~dB}$ between 800 and $2000 \mathrm{~Hz}$ when the knee is upstream. A similar increase is also found in the predictions, mainly due to the change in the mean flow velocity (Table 3) as a result of the differences in the distance to the front of the train. There may also be some changes in the flow interactions between pantograph components for the different orientations, but it is difficult to in- 
clude this in the prediction model. The difference between the predicted and measured OASPL over the frequency range 200 and $4000 \mathrm{~Hz}$ is $0.2 \mathrm{~dB}(\mathrm{~A})$ for knee downstream and $1.3 \mathrm{~dB}(\mathrm{~A})$ for knee upstream.
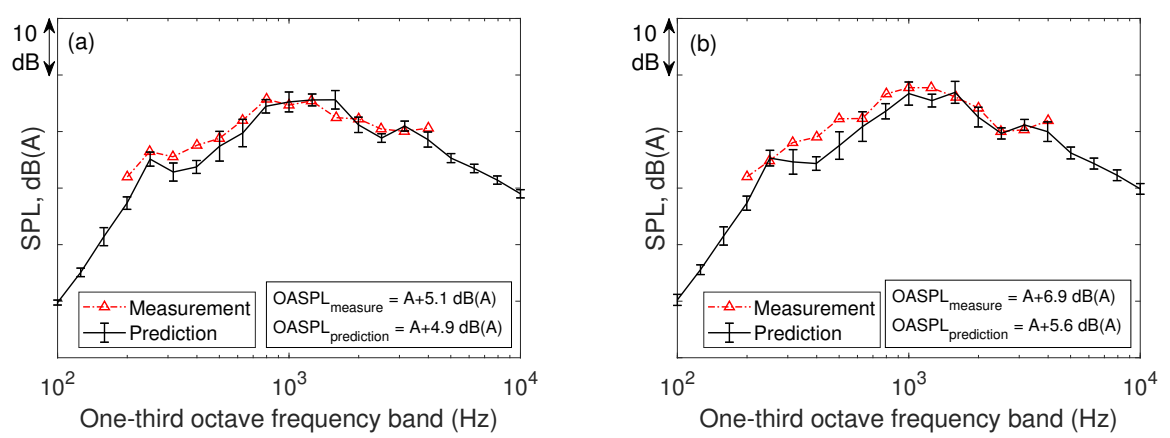

Figure 12: Comparisons between measured and predicted noise spectra of the panhead for pantograph 1 at $273 \mathrm{~km} / \mathrm{h}$. The predicted results are plotted in terms of the mean and a range of $+/$ - one standard deviation (represented by the error bar). (a) Knee joint downstream; (b) Knee joint upstream. Graphs are to the same scale. The OASPL values are shown relative to the measured value for the knee joint (for knee downstream orientation) as in Figure 11

In the predictions, the spectral levels were also calculated for different receiver heights, which affect the directivity angle; the results in terms of the average and the range over the heights are shown in Figure 13. It is seen that the spectral levels around $250 \mathrm{~Hz}$ and $630 \mathrm{~Hz}$ are more sensitive to the receiver height than those at other frequencies. A variation of $7 \mathrm{~dB}$ is found at the peak at $250 \mathrm{~Hz}$ and $4 \mathrm{~dB}$ at $630 \mathrm{~Hz}$. This can be explained by looking at the contributions from individual pantograph components, which are presented in Figure 14 .

Through the prediction model, the contributions from individual pantograph components can be identified. The whole pantograph is divided into 35 components in the model and the spectral levels in different frequency ranges are determined by contributions from a number of components. The components presented in Figure 14 are the most significant ones contributing to the peaks in the spectra. The noise level range due to the receiver height is also presented for the individual components. 

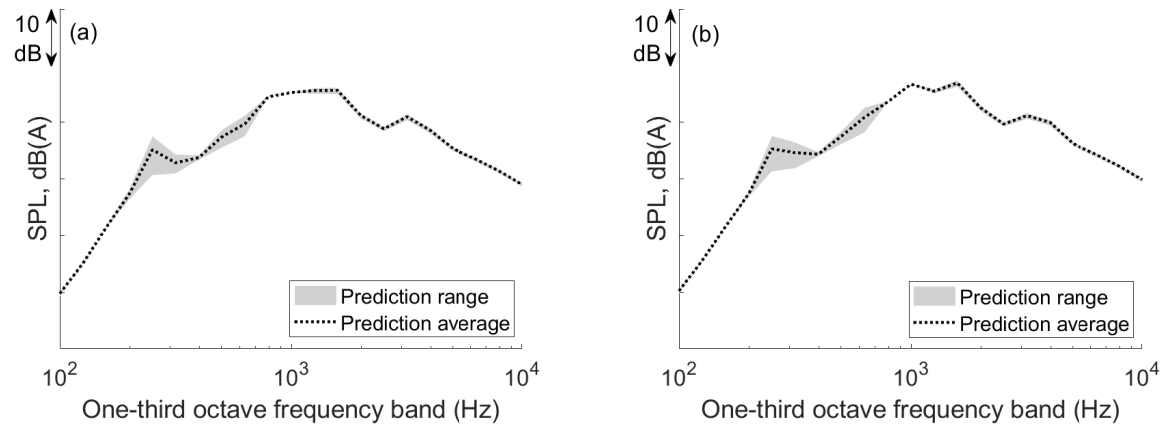

Figure 13: The range of the predicted results found for different heights of microphones in the range -2 to $2 \mathrm{~m}$ relative to the array centre. (a) Knee joint downstream; (b) Knee joint upstream. Graphs are to the same scale.

It is found that the dominant source at the first peak at $250 \mathrm{~Hz}$ is the central segment of the contact strip which also generates high levels of broadband noise over the whole frequency range. The tonal noise from the contact strip radiates mainly in the vertical direction and the directivity angle is sensitive to the receiver height. A $9 \mathrm{~dB}$ variation is found in the peak level of the contact strip. There are also some less important components contributing to this peak, such as the control strut which radiates mainly in the lateral direction and is much less sensitive to the receiver height.

At frequencies around $630 \mathrm{~Hz}$, the horn bush dominates the noise; this radiates in the vertical direction and thus a large variation is seen for the peak. The levels at other frequencies are much less affected by the receiver height because the dominant components radiate in other directions, such as the peaks at 800 and $1250 \mathrm{~Hz}$ which are caused by the horn segments.

\subsection{Results for pantograph 2}

The noise maps for the second pantograph in both orientations are shown in Figure 15. Similar to the first pantograph, the panhead and knee joint are also found to be important sources for both orientations. There are small changes in the sound level of the panhead, which decreases slightly when the knee joint is upstream. The main difference is in the sound level of the knee joint, which is 


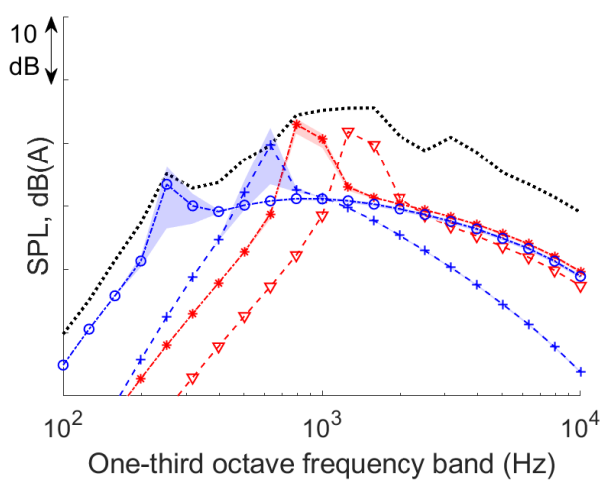

Figure 14: A-weighted noise spectra of selected components of pantograph 1 at $273 \mathrm{~km} / \mathrm{h}$. The shaded regions indicate the prediction range for the individual components due to different receiver heights. ..., panhead; $-\circ$, contact strip; $-\times$, horn bush; $-*$, horn segment 1 and $-\nabla$, horn segment 2 .

much lower than that of the panhead when it is downstream but increases significantly when the knee joint is upstream. For the two orientations, the pantograph is located at different distances from the front of the train. Consequently, the boundary layer thickness at the pantograph location will be different and thus the flow coming towards the pantograph will have different speeds. When this pantograph is in the knee upstream orientation it is quite close to the front of the train (the position is listed in Table 4) where the boundary layer is relatively thin, so the knee joint is likely to be exposed to a higher mean flow velocity than for the downstream orientation.

The measured A-weighted noise spectra for the panhead, knee joint and whole pantograph are presented in Figure 16. For both orientations, the highest levels in the spectrum for the whole pantograph occur at frequencies around 1000 Hz. For the knee downstream, the spectrum is dominated by the panhead over the whole frequency range $400-4000 \mathrm{~Hz}$; the levels of the knee joint are around $10 \mathrm{~dB}$ lower. When the knee is upstream, the levels of the panhead are slightly decreased for frequencies above $630 \mathrm{~Hz}$ whereas the levels for the knee joint are increased by about $6 \mathrm{~dB}$ and become comparable to those for the panhead. The OASPLs for the whole pantograph, the panhead and the knee joint are shown in Figure 16 for both knee orientations. The OASPL of the knee joint for 
Knee-joint downstream

Train running

direction
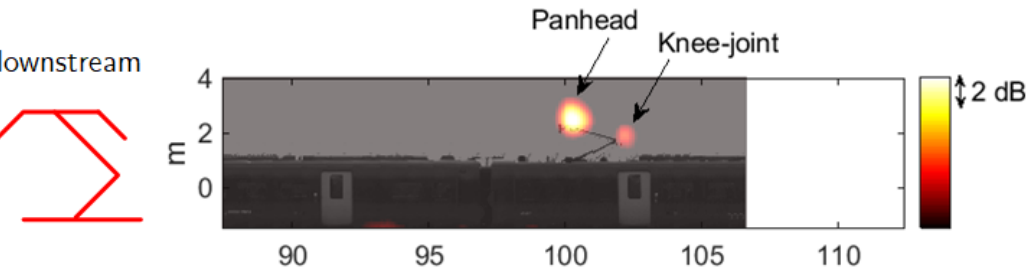

$\mathrm{m}$

Knee-joint upstream

Train running

direction
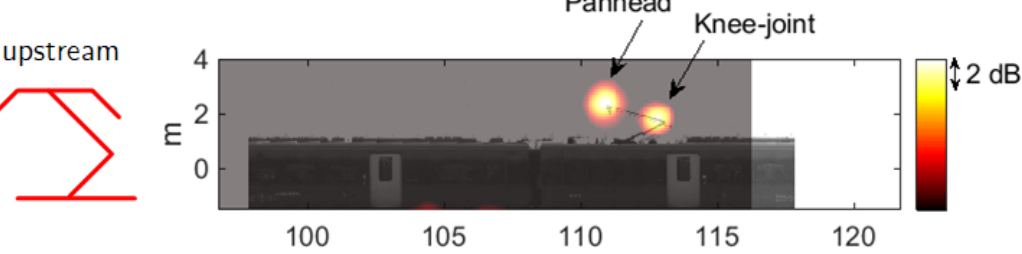

Panhead

$\mathrm{m}$

Figure 15: Noise maps over 400 to $4000 \mathrm{~Hz}$ for pantograph 2 at $226 \mathrm{~km} / \mathrm{h}$ with knee-joint downstream (Top) and upstream (Bottom) (see also in [41). Colour maps are to the same scale.

this pantograph (for knee downstream case) is indicated as $\mathrm{B} d \mathrm{~B}(\mathrm{~A})$; the other OASPLs are shown relative to this value. The OASPL of the whole pantograph for knee upstream is about $1 \mathrm{~dB}(\mathrm{~A})$ higher than that for knee downstream.
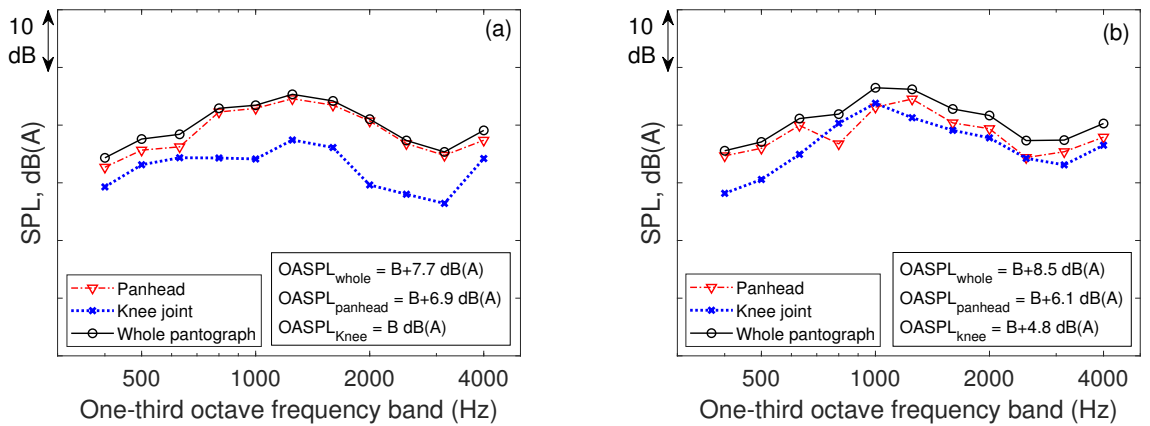

Figure 16: Measured noise spectra of two source regions, the panhead and the knee joint for pantograph 2: (a) knee joint downstream; (b) knee joint upstream. Graphs are to the same scale. OASPL values are shown relative to the value for the knee joint (for knee downstream orientation).

Figure 17 shows comparisons between the predictions and measurements of the noise spectrum from the panhead of pantograph 2 . The predicted results are presented in terms of the mean and a one standard deviation range obtained 
based on 4000 combinations of $U_{0}, I u$ and $L_{x}$. It is seen that both the spectral shape and levels are well predicted by the current model, with a maximum difference of $4 \mathrm{~dB}$ for the knee joint downstream configuration. The agreement with the measurement is also commendable for the knee upstream case although larger deviations are found below $1000 \mathrm{~Hz}$. The difference between the predicted and measured OASPL over 200 and $4000 \mathrm{~Hz}$ is $0.2 \mathrm{~dB}(\mathrm{~A})$ for knee downstream and $1.7 \mathrm{~dB}(\mathrm{~A})$ for knee upstream. Again, although the current prediction model included the effect of the incoming flow conditions for the different orientations, this is unable to account fully for the differences that were found in the measured spectra. Considering the semi-empirical nature of the model and its inherent limitations in capturing all the physics of the noise sources, the predictions for the pantograph noise in realistic conditions are very promising.
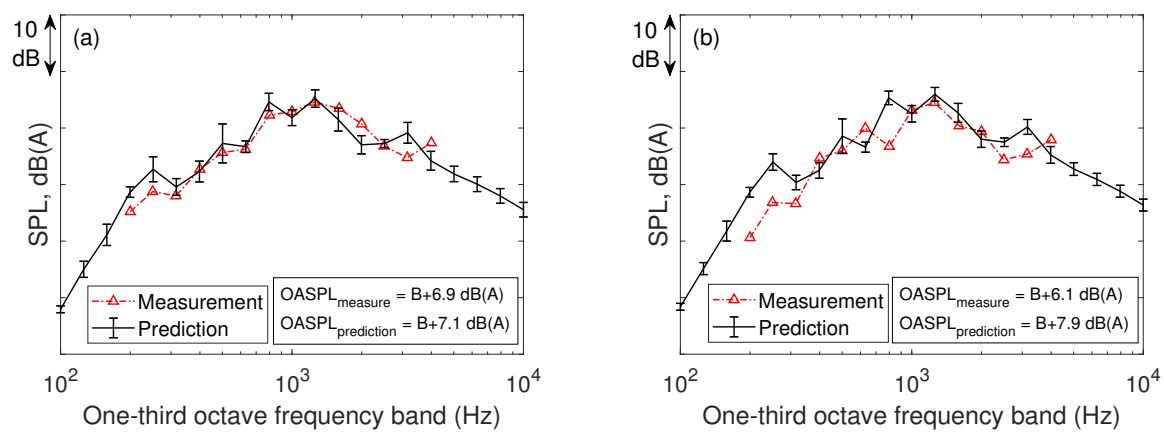

Figure 17: Comparisons between measured and predicted noise spectra of the panhead for pantograph 2 at $226 \mathrm{~km} / \mathrm{h}$. The predicted results are plotted in terms of mean and a range of +/- one standard deviation (represented by the error bar). (a) Knee joint downstream; (b) Knee joint upstream. Graphs are to the same scale. The OASPL values are shown relative to the measured value for the knee joint (for knee downstream orientation) as in Figure 16

Figure 18 shows the sensitivity of the spectral levels to the receiver height for pantograph 2. Compared with pantograph 1 (see Figure 13), similar variations are seen at $250 \mathrm{~Hz}$, while the variation at $630 \mathrm{~Hz}$ is larger as the peak from the horn bush becomes more pronounced due to the lower turbulence intensity level. For a circular component at subcritical Reynolds number, which is the case for the horn bush, the value of $C_{\mathrm{Lrms}}$ as well as the peak amplitude decreases with 
increasing turbulence intensity level (see Section 3.3). Again, the highest noise levels, concentrated in the range $630-2000 \mathrm{~Hz}$, are mainly caused by the horn segments. The contributions from the individual components are similar to those found for pantograph 1 and are not repeated here.
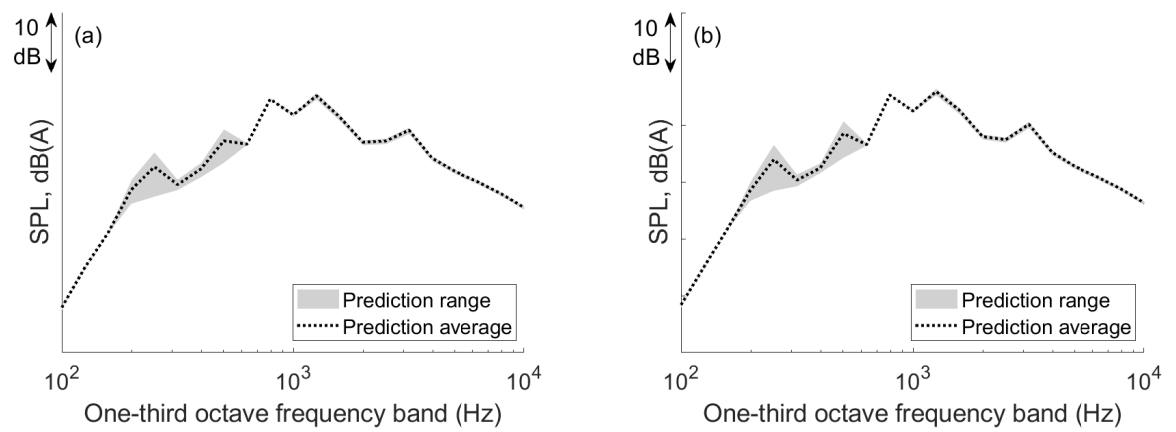

Figure 18: The range of the predicted results found for different heights of microphones in the range -2 to $2 \mathrm{~m}$ relative to the array centre. (a) Knee joint downstream; (b) Knee joint upstream. Graphs are to the same scale.

Figure 19 shows the dependence of the predicted and measured A-weighted OASPL on the train speed for the full pantograph. The measured levels for the knee downstream are generally $1 \mathrm{~dB}(\mathrm{~A})$ lower than those for the knee upstream. The differences between the predicted and measured results are less than 2 $\mathrm{dB}(\mathrm{A})$ for both orientations. The best-fit straight line is also plotted in each case, and from its slope, the speed exponent is shown. The speed exponent is close to 6 in each case, which is typical of dipole-type sources.

\section{Conclusions}

A component-based model for pantograph noise has been extended in the following ways: use is made of results from CFD simulations to determine model constants as well as the Reynolds number dependence, the effect of incoming turbulence is extended to include the subcritical Reynolds number range, and the effect of cylinder aspect ratio on broadband noise amplitude and relative bandwidth is included. With the updated model constants, the model gives 


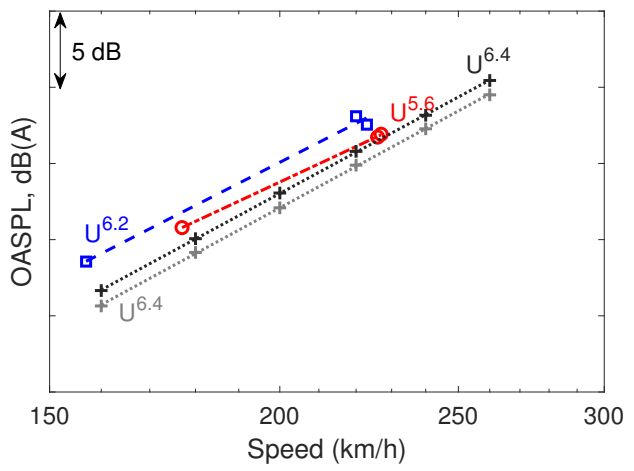

Figure 19: A-weighted measured and predicted OASPL for the whole pantograph for pantograph 2 over 200 to $4000 \mathrm{~Hz}$ as a function of train speed. The speed dependence is calculated separately for the two pantograph orientations. $\square$, measured knee upstream; $\circ$, measured knee downstream; + , predicted knee upstream; + , predicted knee downstream.

better predictions at low frequency in comparison with the wind tunnel measurements. The extension to the effect of incoming turbulence is important for the predictions under realistic incoming flow conditions, which is the case in this paper, as the upstream pantograph components having high turbulence intensity level are still in the subcritical Reynolds number range due to the large incoming turbulence length scale.

The updated model has then been used to predict aerodynamic noise from pantographs in realistic situations during train pass-by. The realistic incoming flow conditions have been estimated from the literature considering the boundary layer development along the train roof. Through Monte Carlo simulations, the prediction results have been obtained in terms of the mean and standard deviation. These results have been compared with field measurements obtained using a microphone array for pantograph on different operational trains. Good agreement is obtained in terms of both the spectral trend and level with a maximum difference in one-third octave bands of less than $4 \mathrm{~dB}(\mathrm{~A})$ over the considered frequency range 200 to $4000 \mathrm{~Hz}$. The differences between the predicted and measured overall level are less than $2 \mathrm{~dB}(\mathrm{~A})$ for both pantographs in both orientations.

The effect of the pantograph orientation was investigated for both pan- 
tographs. From the measurements it was found that the contribution of the knee joint was much smaller than that of the panhead for the first pantograph in both orientations and for the second pantograph with knee downstream. However, for the second one with knee upstream, compared with the knee downstream case, the noise level of the panhead is slightly lower whereas the noise level of the knee joint is larger and becomes comparable to that from the panhead. This large change in the noise level for the knee joint may be related to the fact that, when operating with knee upstream, this pantograph is close to the front of the train where the boundary layer is thinner and so the inflow velocity at the knee joint region is greater. The measured overall levels for knee upstream are about $1 \mathrm{~dB}(\mathrm{~A})$ higher than those for knee downstream for both pantographs. Slight variations in the noise level for different orientations were also found in the predicted results due to the effect of different incoming flow conditions.

The dependence on the operational speed was also investigated for the second pantograph. The speed exponent of the noise level is close to 6 in each case, which is typical of dipole-type sources.

\section{References}

[1] D. Thompson, Railway Noise and Vibration: Mechanisms, Modelling and Means of Control, Elsevier: Oxford, 2008.

[2] N. Curle, The influence of solid boundaries upon aerodynamic sound, Proceedings of the Royal Society of London. Series A. Mathematical and Physical Sciences 231 (1187) (1955) 505-514.

[3] M. J. Lighthill, On sound generated aerodynamically. i. general theory, Proceedings of the Royal Society of London. Series A. Mathematical and Physical Sciences 211 (1107) (1952) 564-587.

[4] D. J. Thompson, E. Latorre Iglesias, X. Liu, J. Zhu, Z. Hu, Recent developments in the prediction and control of aerodynamic noise from high-speed trains, International Journal of Rail Transportation 3 (3) (2015) 119-150. 
[5] C. Talotte, Aerodynamic noise: a critical survey, Journal of Sound and Vibration 231 (3) (2000) 549-562.

[6] K. Nagakura, Localization of aerodynamic noise sources of Shinkansen trains, Journal of Sound and Vibration 293 (3) (2006) 547-556.

[7] B. Barsikow, W. King III, E. Pfizenmaier, Wheel/rail noise generated by a high-speed train investigated with a line array of microphones, Journal of Sound and Vibration 118 (1) (1987) 99-122.

[8] M. M. Boone, N. Kinneging, T. Van Den Dool, Two-dimensional noise source imaging with a T-shaped microphone cross array, The Journal of the Acoustical Society of America 108 (6) (2000) 2884-2890.

[9] C. Mellet, F. Létourneaux, F. Poisson, C. Talotte, High speed train noise emission: Latest investigation of the aerodynamic/rolling noise contribution, Journal of Sound and Vibration 293 (3) (2006) 535-546.

[10] H. Noh, Noise-source identification of a high-speed train by noise source level analysis, Proceedings of the Institution of Mechanical Engineers, Part F: Journal of Rail and Rapid Transit 231 (6) (2017) 717-728.

[11] M. G. Dittrich, M. H. A. Janssens, Improved measurement methods for railway rolling noise, Journal of Sound and Vibration 231 (3) (2000) 595609.

[12] F. Poisson, P. Gautier, F. Letourneaux, Noise sources for high speed trains: a review of results in the TGV case, Noise and vibration mitigation for rail transportation systems, Notes on Numerical Fluid Mechanics and Multidisciplinary Design 99 (2008) 71-77.

725

[13] H. Noh, S. Choi, S. Hong, S. Kim, Investigation of noise sources in highspeed trains, Proceedings of the Institution of Mechanical Engineers, Part F: Journal of Rail and Rapid Transit 228 (3) (2014) 307-322. 
[14] B. He, X. Xiao, Q. Zhou, Z. Li, X. Jin, Investigation into external noise of a high-speed train at different speeds, Journal of Zhejiang University Science A 15 (12) (2014) 1019-1033.

[15] F. R. Grosche, G. E. A. Meier, Research at DLR Göttingen on bluff body aerodynamics, drag reduction by wake ventilation and active flow control, Journal of Wind Engineering and Industrial Aerodynamics 89 (14) (2001) $1201-1218$.

[16] T. Lölgen, Wind tunnel noise measurements on full-scale pantograph models, The Journal of the Acoustical Society of America 105 (1999) 1136.

[17] H. Brick, T. Kohrs, E. Sarradj, T. Geyer, Noise from high-speed trains: Experimental determination of the noise radiation of the pantograph, in: Forum Acusticum, 2011.

[18] W. F. King, E. Pfizenmaier, An experimental study of sound generated by flows around cylinders of different cross-section, Journal of Sound and Vibration 328 (3) (2009) 318-337.

[19] E. Latorre Iglesias, D. J. Thompson, M. G. Smith, Experimental study of the aerodynamic noise radiated by cylinders with different cross-sections and yaw angles, Journal of Sound and Vibration 361 (2016) 108-129.

[20] J. Lee, W. Cho, Prediction of low-speed aerodynamic load and aeroacoustic noise around simplified panhead section model, Proceedings of the Institution of Mechanical Engineers, Part F: Journal of Rail and Rapid Transit 222 (4) (2008) 423-431.

[21] J. E. Ffowcs Williams, D. L. Hawkings, Sound generation by turbulence and surfaces in arbitrary motion, Philosophical Transactions of the Royal Society of London. Series A, Mathematical and Physical Sciences 264 (1151) (1969) 321-342. 
[22] M. Ikeda, T. Mitsumoji, Evaluation method of low-frequency aeroacoustic noise source structure generated by shinkansen pantograph, Quarterly Report of RTRI 49 (3) (2008) 184-190.

[23] M. S. Howe, Acoustics of fluid-structure interactions, Cambridge University Press, 1998.

[24] T. Takaishi, A. Sagawa, C. Kato, Numerical analysis of aerodynamic noise emitted from a pantograph based on non-compact Green's function, Journal of Environment and Engineering 5 (1) (2010) 84-96.

[25] M. Ikeda, T. Mitsumoji, Numerical estimation of aerodynamic interference between panhead and articulated frame, Quarterly Report of RTRI 50 (4) (2009) 227-232.

[26] X. Liu, D. J. Thompson, Z. Hu, Numerical investigation of aerodynamic noise generated by circular cylinders in cross-flow at Reynolds numbers in the upper subcritical and critical regimes, International Journal of Aeroacoustics 18 (2019) 470-495.

[27] X. Liu, Z. Hu, D. J. Thompson, V. Jurdic, Reduction of aerodynamic noise from square bars by introducing spanwise waviness, Journal of Sound and Vibration 435 (2018) 323-349.

[28] Y. Wang, D. Thompson, Z. Hu, Numerical investigations on the flow over cuboids with different aspect ratios and the emitted noise, Physics of Fluids 32 (2) (2020) 025103.

[29] H. Yu, J. Li, H. Zhang, On aerodynamic noises radiated by the pantograph system of high-speed trains, Acta Mechanica Sinica (2012) 1-12.

[30] H. Kim, Z. Hu, D. Thompson, Effect of cavity flow control on high-speed train pantograph and roof aerodynamic noise, Railway Engineering Science 28 (2020) 54-74. 
[31] Y. Zhang, J. Zhang, T. Li, L. Zhang, Investigation of the aeroacoustic behavior and aerodynamic noise of a high-speed train pantograph, Science China Technological Sciences 60 (4) (2017) 561-575.

[32] Y. Yao, Z. Sun, G. Yang, W. Liu, P. Prapamonthon, Analysis of aerodynamic noise characteristics of high-speed train pantograph with different installation bases, Applied Sciences 9 (11) (2019) 2332.

[33] E. Latorre Iglesias, D. J. Thompson, M. G. Smith, Component-based model to predict aerodynamic noise from high-speed train pantographs, Journal of Sound and Vibration 394 (2017) 280 - 305.

[34] C. Norberg, Flow around a circular cylinder: aspects of fluctuating lift, Journal of Fluids and Structures 15 (3-4) (2001) 459-469.

[35] H. Fujita, H. Suzuki, A. Sagawa, T. Takaishi, The Aeolian tone characteristics of a circular cylinder in high Reynolds number flow, in: 5th AIAA/CEAS Aeroacoustics Conference and Exhibit, 1999, p. 1849.

[36] G. Schewe, On the force fluctuations acting on a circular cylinder in crossflow from subcritical up to transcritical reynolds numbers, Journal of Fluid Mechanics 133 (1983) 265-285.

[37] H. Fujita, The characteristics of the Aeolian tone radiated from twodimensional cylinders, Fluid Dynamics Research 42 (1) (2010) 1-25.

[38] J. Cheung, W. Melbourne, Turbulence effects on some aerodynamic parameters of a circular cylinder at supercritical numbers, Journal of Wind Engineering and Industrial Aerodynamics 14 (1-3) (1983) 399-410.

[39] B. Vickery, Fluctuating lift and drag on a long cylinder of square crosssection in a smooth and in a turbulent stream, Journal of Fluid Mechanics 25 (3) (1966) 481-494.

805

[40] J. Zhang, G. Squicciarini, D. J. Thompson, Implications of the directivity of railway noise sources for their quantification using conventional beamforming, Journal of Sound and Vibration 459 (2019) 114841. 
[41] X. Liu, J. Zhang, D. J. Thompson, G. Squicciarini, Z. Hu, M. Toward, D. Lurcock, Comparisons of aerodynamic noise results between computations and experiments for a high-speed train pantograph, in: 13th International Workshop on Railway Noise, Ghent, Belgium, 2019.

[42] T. F. Brooks, W. M. Humphreys, Jr, Effect of directional array size on the measurement of airframe noise components, in: 5th AIAA/CEAS Aeroacoustics Conference and Exhibit, 1999.

[43] C. Baker, The flow around high speed trains, Journal of Wind Engineering and Industrial Aerodynamics 98 (6-7) (2010) 277-298.

[44] C. Baker, S. Dalley, T. Johnson, A. Quinn, N. Wright, The slipstream and wake of a high-speed train, Proceedings of the Institution of Mechanical Engineers, Part F: Journal of Rail and Rapid Transit 215 (2) (2001) 83-99.

[45] M. Sterling, C. J. Baker, S. C. Jordan, T. Johnson, A study of the slipstreams of high-speed passenger trains and freight trains, Proceedings of the Institution of Mechanical Engineers, Part F: Journal of Rail and Rapid Transit 222 (2) (2008) 177-193.

[46] D. Soper, C. Baker, M. Sterling, Experimental investigation of the slipstream development around a container freight train using a moving model facility, Journal of Wind Engineering and Industrial Aerodynamics 135 (2014) 105-117.

[47] H. Schlichting, Boundary layer theory, McGraw-Hill, New York, 1968.

[48] F. White, Viscous Fluid Flow, McGraw-Hill, New York, 1991.

[49] D. Durao, M. Heitor, J. Pereira, Measurements of turbulent and periodic flows around a square cross-section cylinder, Experiments in Fluids 6 (5) (1988) 298-304.

[50] A. Dobre, H. Hangan, Investigation of the three-dimensional intermediate wake topology for a square cylinder at high reynolds number, Experiments in fluids 37 (4) (2004) 518-530. 
[51] P. Sijtsma, Experimental techniques for identification and characterisation of noise sources, in: National Aerospace Laboratory NLR, 2004. 